\title{
Representações e intenção de uso da fitoterapia na atenção básica à saúde
}

\author{
Representations and use intention of phytoterapy \\ in primary health care
}

Caroline da Rosa ${ }^{1}$

Sheila Gonçalves Câmara ${ }^{1}$

JorgeU mberto Béria ${ }^{1}$

${ }^{1}$ Programa de

Pós-Graduação em Saúde Coletiva, Universidade Luterana do Brasil.Av. Farroupilha 8001, prédio 14, sala 228, Bairro São José. 92425-900 Canoas RS. caroliner2007@gmail.com
Abstract The current observational study of investigative characteristics aimed to identify the representations and the use of phytotherapy on the primary health care and the factors related to the intention of use of this therapy. The population for this study was composed by 27 physicians from the Family Health Program in the city of Canoas, Rio Grande do Sul State, Brazil. This quality approach research was developed through a semi structured interviewing process embracing: conceptualization, experiences with phytotherapics and phytotherapy on primary health care. Such topics have brought up subcategories like attitudes, peers perception, control over prescription and intention of use of phytotherapics on primary care. The results have shown that the physicians do not have an institutional knowledge about the subject; a greater intention of use is related to the knowledge of the professionals on this therapeutic modality, based on the belief of its scientific evidence. In order to institutionalize phytotherapy in primary health carea morespread promotion of the studies on scientific evidences is necessary, as well as investments in improvements of professional skills. Thus, the general population will beable to ben efit from phytotherapy asa more accessible alternative to health care.

Key words Phytotherapy, Primary health care, Use intention
Resumo 0 presente estudo observacional, decaráter exploratório, objetivou conhecer as representações e a utilização da fitoterapia na atenção básica à saúdee os fatores relacionadosà intenção de uso dessa terapia. A população em estudo foi composta por 27 médicos do Programa de Saúde da Família no município de Canoas, Rio Grande do Sul, Brasil. A pesquisa de abordagem qualitativa foi realizada através de um roteiro de entrevista semiestruturada abrangendo: conceitualização, experiências com fitoterápicos e fitoterapia na atenção básica. Taistemas originaram subcategorias como atitudes, percepção de pares, controle sobre a prescrição e intenção de uso de fitoterápicosna atenção básica. 0 sresultados demonstraram que os médicos não possuem conhecimento institucionalizado sobre o assunto; maior intenção deuso vincula-seao conhecimento dos profissionais sobre essa modalidade terapêutica, decorrente da crença em sua comprovação científica. Para a institucionalização da fitoterapia na atenção básica, faz-se necessária maior divulgação de estudos acerca da comprovação científica, além de investimentos na capacitação dos profissionais. Dessa forma, a população poderá sebeneficiar da fitoterapia, como uma alternativa mais acessível aos cuidados da saúde.

Palavras-chave Fitoterapia, Atenção básica à saúde, Intenção de uso 
Introdução

A pesar de as plantas medicinais já fazerem parte da cultura popular, nas últimas décadas o interesse pela fitoterapia teve um aumento considerável entre usuários, pesquisadores e serviços de saúde ${ }^{1,2,3}$. Na Declaração de Alma-Ata, em 1978, a Organização Mundial da Saúde (OMS) reconhece que $80 \%$ da população dos países em desenvolvimento utilizam práticas tradicionais nos seus cuidados básicos de saúdee $85 \%$ usam plantas ou preparações destas ${ }^{4}$. Desde então, a OM S tem expressado a sua posição a respeito da necessidade de valorizar a utilização de plantas medicinais no âmbito sanitário e na atenção básica à saúde ${ }^{5,6}$.

No Brasil, a temática foi levantada em diversas oportunidades, como em 1986, na 8a Conferência Nacional de Saúde ${ }^{7}$, quando foi recomendada a introdução das práticas tradicionais de cura popular no atendimento público de saúde. Algumasiniciativas deutilização do conhecimento popular e científico disponível têm demonstrado resultados promissores e visível expansão, como o Projeto Farmácias Vivas da Universidade Federal do Ceará, organizado sob influência das recomendações da OM S acerca do emprego de plantas medicinais nos programas de atenção básica à saúde ${ }^{8}$.

Várias prefeiturastêm implantado programas de fitoterapia aplicados nos serviços públicos de saúde - algumas dessas iniciativas com estrutura bastante sólida e acompanhamento sistemático dos resultados, como é o caso de Vitória $(E S)^{9}$, Curitiba (PR $)^{10}$, cidade do Rio de Janeiro (RJ) $)^{11}$, Ribeirão Preto (SP) ${ }^{12}$ e Itapioca (CE) ${ }^{13}$. A avaliação dessas experiências revela sua importância em termos de perspectiva de redução de custos e algumas questões de ordem técnica que influenciam o desempenho da iniciativa, como treinamento dos profissionais envolvidos e padronização das plantas medicinais deacordo com critérios científicos contemporâneos ${ }^{9.13}$. Além disso, os relatos contribuem para um entendimento maior acerca da integração da sabedoria popular e os resultados clínicos obtidos através da utilização da fitoterapia, refletindo na satisfação de médicos eusuários ${ }^{9}$. Segundo levantamento realizado pelo $\mathrm{M} \mathrm{i-}$ nistério da Saúde em 2004 em todos os municípios brasileiros, verificou-se que a fitoterapia está presente em 116 municípios, contemplando 22 unidades federadas ${ }^{14}$.

A publicação da Política Nacional dePráticas Integrativas e Complementares (PN PIC) no Sistema Ú nico deSaúde (SU S), Portaria no 971 14, de
3 de maio de 2006, foi fruto de anos de ensaios referentes a este tema. A política de caráter nacional recomenda a implantação e a implementação de ações e de serviços no Sistema Ú nico de Saúde (SUS), o que inclui a fitoterapia, com o objetivo de garantir a prevenção de agravos, a promoção e a recuperação da saúde com ênfase na atenção básica à saúde. Além de propor o cuidado continuado, humanizado eintegral em saúde, visa contribuir para o aumento da resolubilidade do sistema com qualidade, eficácia, eficiência, segurança, sustentabilidade, controle eparticipação social ${ }^{15}$.

No caso da utilização da fitoterapia, o profissional médico cumpre papel decisivo. As ações e os conceitos praticados por esse profissional são regularmente interpretados pelo povo como legítimos e adquirem caráter de "verdade"16. É, nesse sentido, considerando os valores culturais, que o posicionamento do médico em relação ao uso de fitoterápicos é aspecto de fundamental importância para a compreensão da utilização de fitoterapia na atenção básica à saúde.

De acordo com Ajzen ${ }^{17}$, na Teoria do Comportamento Planejado, a intenção constitui-seem um forte preditor de realização de determinado comportamento. A intenção, por sua vez, é determinada por três fatores princi pais que interagem entre si: a atitude em relação ao comportamento, a norma subjetiva e a percepção de controle. Considerando o comportamento de utilizar fitoterapia na atenção básica à saúde, é preciso, portanto, avaliar as atitudes dos médicos em relação à fitoterapia, sua percepção acerca do que pensam seus pares sobre o tema e sua percepção de capacitação pessoal para fazer uso dessa terapêutica no contexto da Saúde Pública.

A atitude se conceitualiza como o juízo ou avaliação pessoal acerca das possíveis consequências da realização de determinada ação, e inclui pensamentos, sentimentos e predisposição para realizar o comportamento. A norma subjetiva se define como a pressão social ou influência da percepção subjetiva dos outros sobre a execução da ação específica. N esse sentido, os grupos de referência do indivíduo são os que cumprem papel fundamental. 0 controle consiste na crença acerca da própria capacidade do indivíduo para realizar o comportamento ou controlar situações relacionadas a ele $e^{17,18}$.

Esse modelo, de caráter cognitivo, contribui para a compreensão da propensão dos profissionais em prescrever fitoterápicos no âmbito daatenção básica. N esse sentido, o presenteestudo observacional edecaráter exploratório objetivou conhe- 
cer as representações e a utilização da fitoterapia por partedos médicos queatuam na atenção básica no município deCanoas(RS) e osfatores relacionados à intenção de uso dessa terapia.

\section{Metodologia}

O município de Canoas, com população estimada em de 333.322 habitantes, é a quarta cidade em número de habitantes no Rio Grande do Sul ecaracteriza-se por inexistência absoluta de zona rural ${ }^{19}$. A assistência à saúde, através do Programa de Saúde da Família (PSF), no período da coleta de dados, de maio a agosto de 2006, era realizada em 11 das 25 unidades básicas de saúde (UBS) do município.

A população-alvo são os 31 médicos do PSF de Canoas no atendimento aos usuários do SU S. Todos os médicos foram convidados a participar do estudo, tendo havido quatro recusas. Assim, a população em estudo foi composta por 27 médicos.

A pesquisa de abordagem qualitativa foi desenvolvida através de um roteiro de entrevista semiestruturada. Foi avaliado o desempenho do instrumento em um estudo piloto, realizado no PSF de Porto Alegre (RS), envolvendo três profissionais médicos. 0 roteiro final de entrevista semiestruturada abarca os seguintes temas de interesse: conceitualização, experiências com fitoterápi cos e fitoterapia na atenção básica à saúde. A partir da explanação dos médicos sobre os temas, buscou-se identificar aspectos relacionados a atitudes, norma subjetiva e percepção de controle do profissional, como fatores relacionados à intenção de prescrição. As entrevistas foram realizadas pela pesquisadora, primeira autora deste estudo, no próprio local de trabaIho dos participantes, ou seja, nas UBS, nos consultórios de atendimento. As entrevistas duraram cerca de uma hora eforam gravadas e transcritas para possibilitar a posterior análise dos dados. Os dados referentes à transcrição das entrevistas foram submetidos à análise temática de conteúdo, que consiste em descobrir núcleos de sentido que compõem o texto em análise ${ }^{20}$. A apresentação da falas transcritas respeitou a grafia e a sintaxe utilizadas pelos participantes. Alguns dados foram quantificados em termos de frequência, como sugere Bardin ${ }^{20}$, para melhor caracterizar o contexto deconhecimento e representação dos médicos acerca dos fitoterápicos.

O estudo foi autorizado pela Secretaria M unicipal de Saúde de Canoas. A coordenação do
PSF informou a localização das U BS com equipes do PSF eagendou a coleta de dados. Os princípios éticos foram cumpridos em relação à coleta e à análise dos dados de acordo com as normas de pesquisa em saúde referidas pela Resolução no $196^{21}$ do Conselho Nacional de Saúde.

\section{Resultados ediscussão}

Os 27 médi cos que responderam aosinstrumentos de pesquisa perfazem $87 \%$ da população. A maioria é do sexo masculino (63\%). A faixa etária variou entre 26 e 57 anos, sendo que $63 \%$ dos profissionais médicos possuem até 30 anos. Quanto à formação técnica, 40,7\% são clínicos gerais, 40,7\% possuem especialização em saúde da família e comunidade e $18,6 \%$ outras especialidades médicas.

Quando questionados sobre o uso da fitoterapia, 77,8\% dos participantes afirmam que utilizam na sua vida pessoal e 70,4\% com seus pacientes. Junto a esta informação, foi investigada, também, a última vez em que houve indicação aos pacientes. Com isso, é possível induzir os participantes a buscarem em sua memória um dado concreto. N esse sentido, 40\% utilizaram nos últimos dias, 35\% há al gumas semanas e 25\% há alguns meses ou há mais de um ano.

N a análise das plantas mais utilizadas ou lembradas, identificaram-se a camomila (33,3\%) ea gingko biloba (18,5\%), respectivamente. Sobreas ações terapêuticas, maior frequência foi encontrada para o efeito calmante no tratamento dos sintomas da ansiedade (Tabela 1). Muitas vezes os participantes se valiam do exemplo de dar início ao tratamento terapêutico com plantas medicinais em vez defármacos benzodiazepínicos.

A análise dos dados das entrevistas permitiu maior compreensão acerca das representações dos participantes em relação à fitoterapia. Após realizar a leitura flutuante das entrevistas, foi possível identificar, nos eixos temáticos do estudo, as categorias e as subcategorias sistematizadas no Quadro 1. Os eixos temáticos foram estabelecidos a priori no roteiro de entrevista semiestrurada, exceto o eixo temático denominado cientificidade, que emergiu das respostas dos participantes e se constituiu em um tema recorrente nas entrevistas.

Pela faixa etária dos médicos do PSF do município de Canoas, pode-se concluir que, em sua maioria, são profissionais que saíram da formação superior recentemente. N esse sentido, é possível pensar que a fitoterapia ainda não é parte 
do currículo do curso de medicina de maneira geral. Embora os participantes tenham definido fitoterapia corretamente, esse conhecimento não

Tabela 1. Uso de medicamentos fitoterápicos e/ou plantas medicinais nos agravos em saúde por parte dos médicos do PSF (Canoas, 2006) $(n=27)$.

\begin{tabular}{lc}
\hline \multicolumn{1}{c}{ Ações terapêuticas } & $\%$ \\
\hline Calmante & $29,6 \%$ \\
Estomáquica & $25,9 \%$ \\
Anti-inflamatória & $22,2 \%$ \\
Indutor do sono & $22,2 \%$ \\
Tratamento das afecções do sistema & $14,8 \%$ \\
respiratório & \\
Circulação sanguínea & $14,8 \%$ \\
Circulação periférica & $14,8 \%$ \\
Antisséptico bucal & $11,1 \%$ \\
Tratamento dos sintomas do climatério & $11,1 \%$ \\
Hipocolesterolemiante & $7,4 \%$ \\
Cicatrizante & $7,4 \%$ \\
Outras (hidratação, efeito laxativo, & $3,7 \%$ \\
tratamento das afecções do sistema & \\
urinário, redução da pressão arterial, & \\
hipoglicemiantes) & \\
\hline
\end{tabular}

parece ter vinculação com a academia. Alguns participantes $(37 \%)$ relatam ter tido contato com a fitoterapia no contexto da faculdade, mas nenhum refere ter cursado, durante a sua formação, disciplina queabordasseo tema em seu conteúdo programático. De acordo com Queiroz ${ }^{22}$, a introdução de cursos sobre terapias alternativas em faculdades de medicina é raríssima, embora o relatório da Ciplan (Comissão Interministerial de Planejamento e Coordenação do governo brasileiro) ${ }^{23}$, em 1988, tenha recomendado a inclusão de conhecimento de práticas alternativas no currículo de ensino em saúde.

0 fato de não serem estimulados a essa terapia na formação técnica é ressaltado pelos profissionais que, no exercício da profissão, deparam-se com essa prática através da demanda dos próprios pacientes. As fontes informais de informação (leituras de material não técnico, televisão, contato com outras pessoas, conhecimento geral) foram identificadas como predominantes, já que o movimento dos profissionais parece ser o de uma busca por informações que supram as necessidades com que se defrontam no cotidiano de trabalho na atenção básica à saúde. Para minimizar a distância entre os profissionais de

Quadro 1. Eixos temáticos, categorias e subcategorias do estudo qualitativo.

\begin{tabular}{|c|c|c|}
\hline Eixos temáticos & Categorias & Subcategorias \\
\hline \multirow{3}{*}{ Conceitualização } & Definição & \\
\hline & \multirow[t]{2}{*}{ Origem do conhecimento } & $\begin{array}{l}\text { Conhecimento institucionalizado } \\
\text { Atitudes quanto ao uso } \\
\text { Percepção dos pares (norma subjetiva) }\end{array}$ \\
\hline & & $\begin{array}{l}\text { Fonte informal } \\
\text { Percepção dos usuários (norma subjetiva) }\end{array}$ \\
\hline \multirow{4}{*}{$\begin{array}{l}\text { Experiência com } \\
\text { fitoterápicos }\end{array}$} & Utilização profissional & $\begin{array}{l}\text { Atitudes quanto ao uso } \\
\text { Percepção dos pares (norma subjetiva) }\end{array}$ \\
\hline & Capacitação & Controle sobre o uso \\
\hline & \multirow{2}{*}{ Avaliação } & $\begin{array}{l}\text { Efetividade } \\
\text { Controle sobre o uso }\end{array}$ \\
\hline & & $\begin{array}{l}\text { Aceitação dos usuários } \\
\text { Percepção dos usuários (norma subjetiva) }\end{array}$ \\
\hline \multirow{2}{*}{ Cientificidade } & Comprovação científica & $\begin{array}{l}\text { Atitudes quanto ao uso } \\
\text { Controle sobre o uso }\end{array}$ \\
\hline & Acesso aos estudos & $\begin{array}{l}\text { Atitudes quanto ao uso } \\
\text { Controle sobre o uso }\end{array}$ \\
\hline \multirow{2}{*}{$\begin{array}{l}\text { Fitoterapia } \\
\text { na atenção básica } \\
\text { à saúde }\end{array}$} & Percepção & $\begin{array}{l}\text { Percepção dos pares (norma subjetiva) } \\
\text { Percepção dos usuários (norma subjetiva) }\end{array}$ \\
\hline & Utilização & Intenção de uso \\
\hline
\end{tabular}


saúde e os usuários do SU S, em 2005 foi lançado o Programa Nacional de Reorientação de Formação Profissional em Saúde, o Pró-Saúde ${ }^{24}$. Essa iniciativa integrada do M inistério da Saúde e do Ministério da Educação tem como objetivo a aproximação entre a formação de graduação no país e as necessidades da atenção básica para se construir um fortalecimento do sistema.

Nas entrevistas, a expressão "prescrição" não era utilizada. Os profissionais preferiam termos como recomendar, aconselhar, orientar, utilizar. Assim, somente "recomendam" os fitoterápicos dos quais possuem conhecimento. A utilização profissional dosfitoterápicos necessita estar fundamentada em bases farmacológicas. Essa negação pode ser compreendida pelo fato de a Resolução no $1.499^{25}$, do Consel ho Federal de M edicina, estabelecer a proibição de utilização de terapias não comprovadas pelos modelos reconhe cidos cientificamentena prática médica, incluindo diversas terapias consideradas alternativas. Segundo tal resolução e de acordo com diversos profissionais que a apoiam, é necessário que as terapias alternativastenham sua eficácia demonstrada cientificamente, equando isto ocorrer deixarão de ser alternativas e passarão para o campo médico.

Os participantes expressaram a falta de estudos clínicos comprovando eficácia, indicações, validade de uso, dosagem econtraindicações dos medicamentos fitoterápicos, utilizando repetidamente a expressão "medicina baseada em evidências". A medicina baseada em evidências éidentificada como a norteadora da prática médica atual. A recusa dos procedimentos consagrados pela ciência e adoção do método alternativo significa um perigo de retrocesso em termos de ascensão social, já que implica uma renúncia aos caminhos trilhados pela elite intelectual e uma afirmação de uma proposta que esta elite despreza, ou seja, uma prática considerada pré-científica ${ }^{22}$. A penas um participante ( participante $n^{0}$ 6) afirmou que os estudos que envolvem os medicamentos al opáticos são os mesmos para os medicamentosfitoterápicos. Essas questões estão identificadas nas seguintes falas:

Q uando a gente abre um livro pra consultar a terapia de determinada doença, se tivesse lá uma alternativa fitoterápica, que se enquadrasse dentro dos critérios de saúde adotada na medicina baseada em evidência, talvez houvessealguma predileção por uma ou por outra ( participanteno 14 ).

0 problema maior que eu acho que a gente... como não é nada baseado em evidência, a gente não tem poder de fazer uma prescrição, né? Ou de orientar e dizer: usa isso aqui que faz bem. Até porque na minha visão isto é antiético, né? (participante $n^{\circ}$ 9).

No caso da fitoterapia, os participantes consideram falha a divulgação dos estudos com fitoterápicos entrea classemédica. Referem a possibilidade de que os estudos que comprovem a eficácia científica da fitoterapia sejam realizados, porém eles não têm acesso ou estímulo para buscá-los. De fato, algumas plantas medicinais e medicamentos fitoterápicos foram avaliados, em estudos in vitro e in vivo, na fase clínica e préclínica, contemplando a metodologia da medicina baseada em evidência, muitas com propriedades terapêuticas comprovadas ${ }^{26-29}$. Entretanto, essa diferenciação não aparece nas entrevistas, indicando que, realmente, 0 acesso às informações é bastante restrito. Isso também porque essa área abrange não somente a medicina baseada em evidências, mas também uma concepção distinta sobre o fenômeno saúde-doença, o que inclui a interação do saber popular com os conhecimentos científicos ${ }^{30}$.

Dessa forma, percebe-se uma atitude desfavorável dos profissionais quanto ao uso de fitoterápicos que vem reforçada pelas representações mais amplas da classe médica. Ao se verem entre as práticas dos usuários e a comprovação científica, os médicos fazem seu julgamento em relação ao uso de fitoterápicos e há pouco questionamento acerca de suas propriedades cientificamente comprovadas. A pressão de grupo, aqui compreendida como norma subjetiva, apresenta-se como uma importante pauta de atuação do médico. Pauta esta que surge como um questionamento acerca da própria credibilidade da atuação dos profissionais no campo da atenção básica.

A preocupação dos participantes sobre este saber que eles ainda não dominam pode ser entendida como um fator que dificulta a inserção da fitoterapia como rotina no atendimento à população.

0 médico vai ter que reestruturar, vai ter que aprender a mexer nos fitoterápicos, vai ter que ver os resultados. E isso demora de três a cinco anos pra fazer. Eu não uso o meu paciente como cobaia, eu recuso isso (participante $\mathrm{n}-20$ ).

Então eu prefiro não me aventurar num campo que em que eu não tô familiarizado, esse é 0 principal motivo da minha não adoção da prescrição, mais por falta de conhecimento a respeito do que propriamente por não acreditar que ele tenha um efeito. $\mathrm{Ou}$ melhor, tanto acredito que eles tenham um efeito que não me aventuro a prescrevê 
lospor medo queinterajam com alguma outra droga que a pessoa esteja usando (participante $n-14$ ).

$M$ as enquanto eu não fizer um curso, alguma coisa, né?, que me dê capacidade pra prescrever isso, eu não vou prescrever assim num receituário enquanto não me sentir capacitado pra isso (participanten-26).

A falta de conhecimento dos profissionais de saúde sobre a utilização das terapias alternativas e, mais ainda, do sistema de interpretação dos sinais e dos sintomas utilizados popularmente é fato reconhecido tanto por eles como pela população. 0 conhecimento das doenças que o médico não cura e de certos medicamentos que o mé dico não receita identifica este contexto já institucionalizado das diferenças entre a medicina oficial e a paralela ${ }^{31}$.

A percepção de pouca eficácia para a utilização de fitoterápicos na prática cotidiana remete diretamente à percepção de controle do médico quanto a esta terapia. 0 controleestá diretamente relacionado ao conhecimento que se dispõe sobre o tema, enesse sentido os participantes apresentam uma postura ética em razão do seu receio em explorar campos pouco conhecidos ou divulgados em seus âmbitos de referência como classe profissional.

Os médicos que participaram deste estudo reconhecem que a população tem boa aceitação por essa terapia - fato também já descrito pela $\mathrm{OM} \mathrm{S}^{32}$, quando afirmou que a fitoterapia constitui uma prática "que emana do povo e é bem aceita por este". Tal aspecto éabordado na Portaria $\mathrm{n}$ - $971^{14}$, quando diz que a fitoterapia "incentiva o desenvolvimento comunitário, a solidariedadeea participação social". Essa percepção, acerca da aceitação dos usuários, é relacionada pelos participantes à utilização da fitoterapia na atenção básica à saúde. Ocorre uma vinculação da fitoterapia como elemento da cultura popular envolvendo a crença no poder de cura.

...porque tem muito de fé junto também, eu acho, né?! A s pessoas vão atrás... D esdequemel hore, não importa de que jeito (participante no 26).

É o vínculo com a medicação fitoterápica no sentido de uma crença, de uma ajuda a mais ( participante $n^{0} 19$ ).

... até porque eu acho que a fitoterapia envolve algo mais que prescrever medicamentos. Também envolve um outro tipo de abordagem, a compreensão de doenças etc. (participante $n 0$ - 4).

A utilização dessa prática na rotina de atendimento do serviço de saúde é relacionada pelos participantes deste estudo a fatores que contribuiriam para uma atenção básica mais efetiva, cumprindo a sua função primordial, a promoção da saúde, como a valorização da prática de autocuidado e a aproximação da relação médico-paciente. Além disso, uma possível vantagem em termos de redução de custos, apontada pelos participantes, fornece subsídios para o fortalecimento dessas opiniões favoráveis à inserção dessa terapia para promover a cura ea prevenção de doenças dentro do sistema.

É um meio menosdispendioso para o paciente, que resgata uma cultura de autocuidado junto... (participante no 24).

Seria o local perfeito pra ela, o melhor local de atuação: atenção primária à saúde; reduziria custos de medicamentos, levaria ao paciente uma melhor qualidade de vida, a gente puxaria os pacientes mais para nós... (participante $n-4$ ).

Eu tenho certeza que pra rede básica seria ótimo porque além do baixo custo é uma coisa quea população recebe mel hor do que ter que tomar remédio (participante $\mathrm{n} 025$ ).

Dentre os participantes, $77,8 \%$ informaram que utilizam a fitoterapia na vida pessoal - percentual este bastante semel hanteao divulgado na Declaração de Alma-Ata 4 , em 1978, e em outros estudos ${ }^{33,34}$, o que ressal ta a relevância dessa prática no cotidiano dos brasileiros.

A promoção da saúde através de plantas medicinais envolve valores culturais historicamente construídos ${ }^{4}$, e pode e deve ser vivenciada dentro do serviço de saúde ${ }^{14}$, proporcionando, entre outras vantagens, uma aproximação do usuário com o sistema. Observa-se que a história do processo de institucionalização das práticas integrativas e complementares tem sido de muitos percal çosna última década no Brasil, tendo seu percurso encontrado obstácul os constantes interpostos pela medicina social mente hegemônica35. Portanto, não basta os governos instituírem, nos sistemas oficiais, a medicina alternativa ou mesmo a promulgação legal para garantir a sua oferta com qualidade; faz-se necessária a promoção de espaços de discussão tanto no âmbito acadêmico quanto nos serviços, considerando as dificuldades para o uso de um "novo" paradigma de cuidar ${ }^{36}$.

\section{Conclusões econsiderações finais}

0 contexto e o momento da pesquisa mostraramse bastante favoráveis a este estudo. A pesquisa foi projetada antes da publicação da Portaria no $971^{14}$, de 3 de maio de 2006, porém a coleta de dados se iniciou quando haviam passado onze dias da sua 
publicação, momento em que entidades ea sociedade estavam manifestando-se sobre a referida portaria. Turner, citado por Queiroz ${ }^{22}$, afirma que, em uma situação de transição, os valores perdem a sua condição de absolutos, ea realidade passa a ser construída, interpretada, manipulada, destruída e reconstruída, tendo como matéria-prima o confronto de valores múltiplos provenientes da situação vividaindividual ou coletivamentepelosatores sociais. Desse modo, princípios culturais que normalmente não vêm à tona tornam-se explícitos e aparentes para 0 investigador.

Os resultados demonstraram uma representação correta dos médicos acerca do que são os fitoterápicos; no entanto, esseconhecimento também parece ter suas raízes na cultura brasileira e, portanto, nas vivências e no cotidiano de grande parte dos brasileiros. M as a cultura popular ainda é relegada a um segundo plano ante o saber científico, o que cria um contexto de pouco conhecimento científico e abertura a novas possibilidades e insegurança reforçada pelo grupo de pares que defineecaracteriza a profissão médica. Tais representações, derivadas de uma compreensão clássica de ciência, contribuem para a formação de atitudes negativas ou receosas antetecnologias milenares e de fácil acesso, como é o caso dos fitoterápicos. Essa conjunção defatores relaciona-se diretamenteà intenção da utilização de fitoterápicos na atenção básica à saúde.

As práticas enraizadas na sabedoria popular e járeconhecidasno meio científico devem ser integradas, porém de maneira a ser vinculadas à assistência à saúde como um todo. Um sistema de saúde que adota a fitoterapia deve incorporar um conjunto de atitudes, valores e crenças que constituem uma filosofia de vida, e não estabelecer-se como uma mera distribuição demedicamentos ${ }^{30}$.
O SUS resgatou as discussões acerca da humanização e universalização da aten ção à saúde nos serviços oficiais, assim como 0 atendimento integral e a participação da comunidade. Desse modo, possibilitou ao usuário o acesso democrático ea escolha da terapêutica preferida, além de criar uma tensão entre a diversidade de saberes: de um lado, os conceitos científicos atuais sobre fitoterápicos e, de outro, o saber popular sobre $o$ uso das plantas dentro de sua conceitualização de saúde- doen ça ${ }^{11}$. Entre os participantes deste estudo, verificou-se maior intenção de uso da fitoterapia entre aqueles que detêm maior conhecimento sobre o assunto. Esta constatação revela a importância da capacitação dos profissionais e do investimento em programas de educação permanente, com vistas a proporcionar sucesso nessa iniciativa. 0 desenvolvimento de material técnico, incluindo os estudos pré-clínicos e clínicos e a validade de uso das plantas medicinais e/ou medicamentos fitoterápicos selecionados para compor um programa, contribuirá para amenizar a preocupação expressada pelos médicos com os critérios científicos contemporâneos dessa terapia. E a convergência das respostas dos participantes, apontando para as vantagens da inserção dessa prática na aten ção básica à saúde, revela-se como um possível indicador de aceitabilidade da utilização dessa terapia no SUS pelos profissionais médicos.

O uso da fitoterapia na atenção básica à saúde talvez representemais queuma diminuição de custos, pois implica a aceitação do saber do outro, do usuário. Implica o vínculo e o respeito por valores culturais e condições de vida. Pode ser o resultado de uma parceria que rompe com a dicotomia entre os sistemas formal e informal de saúde ${ }^{37}$.

\section{Colaboradores}

C Rosa idealizou a pesquisa e foi a responsável pela realização das entrevistas, análise e discussão dos resultados, tendo planejado e redigido 0 artigo; SG Câmara orientou as análises e a discussão dos resultados, planejou e redigiu 0 artigo; e JU Béria participou do planejamento da pesquisa e também redigiu 0 artigo. 


\section{Referências}

1. World Health Organization (WHO). Legal status of traditional medicine and complementary/alternative medicine. Geneva: World Health Organization; 2001.

2. Hufford DJ. Folk medicine and health culture in contemporary society. Prim Care 1997; 24(4):723-741.

3. Kenny E, Muskin PR, Brown R, Gerbarg PL. What the general psychiatrist should know about herbal medicine. Curr Psychiatry Rep 2001; 3(3):226-234.

4. Organização M undial da Saúde (OM S). Alma-Ata 1978 - Cuidados primários de saúde. Relatório da conferência internacional sobre cuidados primários de saúde. Brasília: Organização M undial da Saúde/Fundo das Nações Unidas para a Infância; 1979.

5. Organización M undial de la Salud (OMS). Consejo Ejecutivo. M edicina tradicional y asistencia sanitaria moderna. Foro mundial de la salud. Revista Internacional de Desarrollo Sanitario 1991; 12(1):120.

6. Organización M undial de la Salud. Estrategia de la OM S sobre medicina tradicional 2002-2005. Geneva: Organización Mundial de la Salud; 2002.

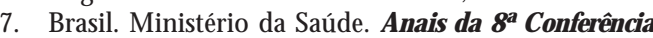
Nacional de Saúde. Brasília: M inistério da Saúde; 1987.

8. Matos FJA. Farmácias vivas: sistema de utilização de plantas medicinais projetado para pequenas comunidades. Fortaleza: EUFC; 1998.

9. Sacramento HT. O programa de fitoterapia do município de Vitória-ES. Divulgação em Saúde para Debate 2004; 30:59-65.

10. Graça C. Treze anos de fitoterapia em Curitiba. Divulgação em Saúde para Debate 2004; 30:36-41.

11. Reis M CP, Leda PHO, Pereira M TCL, Tunala EAM Experiência na implantação do programa de fitoterapia do município do Rio de Janeiro. Divulgação em Saúde para Debate 2004; 30:42-49.

12. Pires AM, Borella JC, Raya LC. Prática alternativa de saúde na atenção básica da rede SUS de Ribeirão Preto-SP. Divulgação em Saúde para D ebate 2004; 30:56-58.

13. Carneiro SMC, Pontes $L M L$, Gomes Filho VAF Guimarães MA. Da planta ao medicamento: experiência da utilização da fitoterapia na atenção primária à saúde no município de Itapioca-CE. Divulgação em Saúde para Debate 2004; 30:50-65.

14. Brasil. Portaria no 971. Aprova a Política Nacional de Práticas Integrativas e Complementares (PNPIC) no Sistema Ú nico de Saúde. Diário Oficial da U nião 2006; 4 maio.

15. Barros NF. Política Nacional de Práticas Integrativas e Complementares no SUS: uma ação de inclusão. Cien Saude Colet 2006; 11(3):850.

16. Vasconcelos EM. A terapêutica médica e as práticas populares de saúde. Saúde em Debate 1996; 49/ 50:101-106.

17. Ajzen I. The theory of planned behavior. Organ Behav Hum Decis Process 1991; 50:179-211.

18. Rubio JM L, Anzano SM. Psicologia social de la salud: fundamentos teóricos y metodológicos. Sevilla: Comunicación Social Ediciones y Publicaciones; 2002.

19. Instituto Brasileiro de Geografia e Estatística (IBGE). Estimativa das populações dos municípios de 2006. [acessado 2007 jun 19]. Disponível em: http:// www.ibge.gov.br/home/estatistica/populacao/estimativa2006/estimativa.shtm

20. Bardin L. Análise de conteúdo. Lisboa: Edições 70; 1977.
21. Brasil. Resolução no 196. Diretrizes e normas regulamentadoras de pesquisa em seres humanos. Brasília: Conselho Nacional de Saúde; 1996.

22. Queiroz MS. 0 itinerário rumo às medicinas alternativas: uma análise em representações sociais de profissionais da saúde. Cad Saude Publica 2000; 16(2):363-375.

23. Resolução Ciplan (Comissão Interministerial de Planejamento e Coordenação) no 8. I mplanta a prática da fitoterapia nos serviços de saúde. Diário Oficial da União 1988; 11 mar.

24. Brasil. Portaria Interministerial n' 2.101. Institui 0 Programa Nacional de Reorientação de Formação Profissional em Saúde - Pró-Saúde - para os cursos de graduação em Medicina, Enfermagem e Odontologia e Enfermagem. Diário Oficial da União 2005; 4 nov.

25. Conselho Federal de Medicina. Resolução № 1.499. Proíbe aos médicos a utilização de práticas terapêuticas não reconhecidas pela comunidade científica. Diário Oficial da União 1998; 3 set.

26. Blumenthal M, Busse WR, Goldberg A, editors. The complet German comission E monographs: therapeutic guide to herbal medicines. Austin: American Botanical Council; 1998.

27. World Health Organization. WHO monographs on selected medicinal plants. Geneva: World Health Organization; 1999.

28. World Health Organization. WHO monographs on selected medicinal plants. Geneva: World Health Organization; 2002.

29. European Scientific Cooperative on Phytotherapy. M onographs on the medicinal uses of plant drugs. Exeter: European Scientific Cooperative on Phytotherapy; 2003.

30. Leite SN. Além da medicação: a contribuição da fitoterapia para a Saúde Pública [dissertação]. São PauIo: Faculdade de Saúde Pública, Universidade de São Paulo; 2000.

31. Loyola MA. M édicos e curandeiros: conflito social e saúde. São Paulo: Difel; 1984.

32. Organización Mundial de la Salud (OMS). Promoción y desarrollo de la medicina tradicional. Geneva: Organización Mundial de la Salud; 1978.

33. Teixeira ER, Nogueira JF. 0 uso popular das ervas terapêuticas no cuidado com o corpo. Rev Gaúcha Enferm 2005; 26(2):231-241.

34. Gomes DLS, Romanholi LM, Souza MTG. A fitoterapia e a homeopatia como práticas médicas alternativas. Rev Bras Enferm 1985; 38(3/4):329-348.

35. Luz MT. Culturas contemporâneas e medicinas alternativas: novos paradigmas em saúde no fim do século XX. Physis 2005; 15(Supl.):145-176.

36. Souza IM C, Vieira AIC. Serviços públicos de saúde e a medicina alternativa. Cien Saude Colet 2005; 10(Supl.):255-266.

37. Saforcada E. Perspectiva ecológico-sistémica de la salud. In: Saforcada E, Sarriera JC, organizadores. Enfoques conceptuales y técnicos em psicología comunitaria. Buenos Aires: Paidós; 2008. p. 49-74.

Artigo apresentado em 18/12/2007

Aprovado em 11/08/2008

Versão final apresentada em 11/09/2008 